\title{
Cooper pairs in atomic nuclei
}

\author{
G. G. Dussel, ${ }^{1}$ S. Pittel, ${ }^{2}$ J. Dukelsky, ${ }^{3}$ and P. Sarriguren ${ }^{3}$ \\ ${ }^{1}$ Departamento de Fisica J. J. Giambiagi, Universidad de Buenos Aires, 1428 Buenos Aires, Argentina \\ ${ }^{2}$ Bartol Research Institute and Department of Physics and Astronomy, University of Delaware, Newark, Delaware 19716, USA \\ ${ }^{3}$ Instituto de Estructura de la Materia, CSIC, Serrano 123, E-28006 Madrid, Spain
}

(Received 6 April 2007; published 5 July 2007)

\begin{abstract}
We consider the development of Cooper pairs in a self-consistent Hartree-Fock mean field for the even Sm isotopes. Results are presented at the level of a BCS treatment, a number-projected BCS treatment and an exact treatment using the Richardson ansatz. For a fixed value of the pairing strength, projected BCS captures much of the pairing correlation energy that is absent from BCS, but still misses a sizable component, typically of order $1 \mathrm{MeV}$. Furthermore, because it does not average over the properties of the fermion pairs, the exact Richardson solution permits a more meaningful definition of the Cooper wave function and of the fraction of pairs that are collective.
\end{abstract}

DOI: 10.1103/PhysRevC.76.011302

The first breakthrough in the derivation of a microscopic theory of superconductivity was the demonstration by Cooper [1] in 1956 that bound pairs could be produced in the vicinity of the Fermi surface for an arbitrarily small attractive interaction. This was followed soon thereafter by the development of the BCS theory [2], in which superconductivity was described as the condensation of a set of correlated pairs averaged over the whole system. Soon after the BCS paper, Bohr, Mottelson, and Pines [3] suggested that a similar phenomenon could explain the large gaps in the spectra of even-even nuclei. Since then, the BCS theory has been widely used to describe superconductivity in condensed matter and nuclear systems. Moreover, the concept of Cooper pairs as strongly overlapping objects that go through a condensation process at the superconducting transition is central in the interpretation of the superconducting phenomenon. However, it is not easy to define the Cooper pair wave function from the mean field BCS theory, and most frequently it has been related to the pair correlator.

By using the exact solution of the BCS Hamiltonian given by Richardson in the 1960s [4], it was recently shown [5] that the Cooper pair wave function in a superconducting medium has a precise definition. The unique form of its wave function transforms from a Cooper resonance in the weak coupling BCS region to a quasibound pair in the Bose-Einstein condensed (BEC) phase. Moreover, the Richardson solution gives a clear prescription for evaluating the fraction of correlated pairs as compared with Yang's definition [6], providing a more accurate description of the condensation phenomenon.

The subject of Cooper pairing in atomic nuclei has come under renewed focus recently in the context of relativistic [7] and nonrelativistic mean-field treatments [8]. Here too we explore the role of Cooper pairs in mean-field treatments of atomic nuclei, comparing the traditional numbernonconserving BCS approach with a projected BCS approach and the exact Richardson treatment. We show that substantial differences in correlation energies arise when pairing is treated exactly for the same pairing strength, and that interesting differences emerge in some conceptual properties of the paired system.
PACS number(s): 21.60.-n, 02.30.Ik, 03.75.Ss, 74.20.Fg

We begin by detailing the differences between the three approaches, focusing on a pairing Hamiltonian with constant strength $G$ acting in a space of doubly-degenerate timereversed states $(k, \bar{k})$,

$$
H=\sum_{k} \epsilon_{k} c_{k}^{\dagger} c_{k}-G \sum_{k, k^{\prime}} c_{k}^{\dagger} c_{\bar{k}}^{\dagger} c_{\bar{k}^{\prime}} c_{k^{\prime}},
$$

where $\epsilon_{k}$ are the single-particle energies for the doublydegenerate orbits $k, \bar{k}$.

Cooper considered the addition of a pair of fermions with an attractive pairing interaction on top of an inert Fermi sea (FS). He showed that the pair eigenstate is

$$
\left|\Psi_{\text {Cooper }}\right\rangle=\sum_{k>k_{F}} \frac{1}{2 \epsilon_{k}-E} c_{k}^{\dagger} c_{\bar{k}}^{\dagger}|F S\rangle,
$$

where $E$ is the energy eigenvalue. It turns out that $E$ is negative for any attractive value of $G$, implying that the Cooper pair is bound and that the FS is unstable against the formation of bound pairs. Cooper suggested [1] that a theory considering a collection of bound pairs on top of an effective FS could explain superconductivity.

The BCS approach follows a somewhat different path, defining instead a variational wave function as a coherent state of pairs properly averaged over the whole system,

$$
\left|\Psi_{\mathrm{BCS}}\right\rangle=e^{\Gamma^{\dagger}}|0\rangle,
$$

where $\Gamma^{\dagger}=\sum_{k} z_{k} c_{k}^{\dagger} c_{\bar{k}}^{\dagger}$ is the coherent pair. Though errors due to the nonconservation of particle number in Eq. (3) are negligible in the thermodynamic limit, they can be important in finite systems such as atomic nuclei, as first pointed out by Bohr, Mottelson, and Pines [3]. To accommodate these effects, number-projected BCS (PBCS) [9] considers a condensate of pairs of the form

$$
\left|\Psi_{\mathrm{PBCS}}\right\rangle=\left(\Gamma^{\dagger}\right)^{M}|0\rangle,
$$

where $M$ is the number of pairs and $\Gamma^{\dagger}$ has the same form as in BCS. We would like to emphasize here that $\Gamma^{\dagger}$ should not be 
confused with the operator that creates a Cooper pair since its structure contains an average over the correlated pairs close to the Fermi energy and the free fermions deep inside the Fermi sphere.

The Richardson ansatz [4] for the exact solution of the pairing Hamiltonian (1) follows closely Cooper's original idea. For a system with $2 M$ particles, it involves (in the $v=0$ sector) a product of $M$ distinct pairs of the form

$$
|\Phi\rangle=\prod_{\alpha=1}^{M} \Gamma_{\alpha}^{\dagger}|0\rangle, \quad \Gamma_{\alpha}^{\dagger}=\sum_{k} \frac{1}{2 \epsilon_{k}-e_{\alpha}} c_{k}^{\dagger} c_{\bar{k}}^{\dagger} .
$$

The $e_{\alpha}$, called pair energies in analogy with the Cooper wave function (2), are in general complex parameters, which are obtained by solving the set of coupled nonlinear Richardson equations

$$
1-G \sum_{k} \frac{1}{2 \epsilon_{k}-e_{\alpha}}-2 G \sum_{\beta(\neq \alpha)=1, M} \frac{1}{e_{\beta}-e_{\alpha}}=0 .
$$

The energy eigenvalues are obtained by summing the lowest $M$ pair energies of each independent solution $\left(E=\sum_{\alpha} e_{\alpha}\right)$.

The key point to note upon inspection of the Richardson pair (5) is that a pair energy close to a particular $2 \epsilon_{k}$, i.e., close to the energy of an unperturbed pair, is dominated by this particular configuration and thus defines an uncorrelated pair. In contrast, a pair energy lying sufficiently far away in the complex plane produces a correlated Cooper pair.

As mentioned before, the BCS coherent pairs, with amplitudes $z_{k}=v_{k} / u_{k}$, cannot be interpreted as Cooper pairs since they mix correlated and uncorrelated pairs over the whole system. Indeed, it has been shown [5] that only in the extreme BEC limit are all pairs bound and condensed and amenable to description by the two approaches. Usually the structure of the Cooper pair is assigned to the pair correlator $\left\langle\mathrm{BCS}\left|c_{k}^{\dagger} c_{k}^{\dagger}\right| \mathrm{BCS}\right\rangle=u_{k} v_{k}$. However, if the BCS state represents a fraction of correlated pairs within a Fermi sea of free uncorrelated fermions, the pair correlator cannot guarantee that it picks up the two fermions from the same pair. The pair correlator is another averaged property over the set of correlated pairs.

In what follows we explore the structure of pairing correlations in the even $\mathrm{Sm}$ isotopes, from ${ }^{144} \mathrm{Sm}$ through ${ }^{158} \mathrm{Sm}$. The results are based on a series of self-consistent deformed Hartree Fock+BCS calculations. The calculations make use of the density-dependent Skyrme force, SLy4, and treat pairing correlations using a pairing force with constant strength $G$.

The calculations are carried out in an axially symmetric harmonic oscillator space of 11 major shells (286 doublydegenerate single-particle states). This basis involves oscillator parameters $b_{0}$ and axis ratio $q$, optimized in order to minimize the energy in the given space. The strength of the pairing force for protons and neutrons is chosen in such a way as to reproduce the experimental pairing gaps in ${ }^{154} \mathrm{Sm}\left(\Delta_{n}=\right.$ $\left.0.98 \mathrm{MeV}, \Delta_{p}=0.94 \mathrm{MeV}\right)$, extracted from the binding energies in neighboring nuclei. We obtain $G_{n}=0.106 \mathrm{MeV}$ and $G_{p}=0.117 \mathrm{MeV}$. Once we have fitted this reference strength, we determine the pairing strengths appropriate to the ${ }^{142-158} \mathrm{Sm}$ isotopic chain by assuming a $1 / A$ dependence. These calculations provide an excellent description of the properties of the even $\mathrm{Sm}$ isotopes.

We then use the results at self-consistency to define the HF mean field and consider the alternative number-conserving PBCS and exact Richardson approach to treat the pairing correlations within this mean field. We ignore the issue of whether the mean field should be self-consistently modified in these other approaches. In this way we are able to directly compare the three approaches to pairing with the same pairing Hamiltonian, which is the focus of this investigation.

As is well known, the numerical solution of the Richardson equations (6) involves instabilities due to singularities arising at critical values of the pairing strength $G$. There have been two recent works that study these critical regions of parameter space [10] and propose ways to overcome the singularities [11]. While these methods alleviate the numerical divergences, thus allowing for interpolation across the critical regions, some problems still persist and we have thus chosen to use a different approach. Since the singularities arise as crossings of real pair energies $e_{\alpha}$ with the unperturbed single-pair energies $2 \epsilon_{k}$ in the denominators of Eq. (6), we start the numerical procedure at strong coupling $(G=1 \mathrm{MeV})$ with complex single-particle energies, obtained by adding a small arbitrary imaginary component. In this way, the singularities are avoided in the evolution of the system from strong coupling almost to the $G=0$ limit. To obtain the exact solution at the physical value of $G$, we then let the imaginary parts go to zero starting with the solution already obtained for that $G$ value. The method seems to work for any distribution of single-particle energies.

A principal focus of our investigation is on the pairing correlation energy, defined as

$$
E_{C}=\left\langle\Phi_{\text {corr }}|H| \Phi_{\text {corr }}\right\rangle-\left\langle\Phi_{\text {uncorr }}|H| \Phi_{\text {uncorr }}\right\rangle,
$$

where $\left|\Phi_{\text {corr }}\right\rangle$ is the correlated ground-state wave function and $\left|\Phi_{\text {uncorr }}\right\rangle$ is the uncorrelated Hartree-Fock-Slater determinant obtained by filling all levels up to the Fermi energy. This quantity reflects the additional energy that derives from the inclusion of pairing.

Table I summarizes our results for the pairing correlation energy for all the even Sm isotopes under consideration. The

TABLE I. Pairing correlation energies associated with the BCS, PBCS, and exact Richardson treatments of pairing for the even $\mathrm{Sm}$ isotopes. All energies are given in $\mathrm{MeV}$.

\begin{tabular}{lccc}
\hline \hline Mass & $E_{C}($ Exact $)$ & $E_{C}(\mathrm{PBCS})$ & $E_{C}(\mathrm{BCS})$ \\
\hline 142 & -4.146 & -3.096 & -1.107 \\
144 & -2.960 & -2.677 & 0. \\
146 & -4.340 & -3.140 & -1.384 \\
148 & -4.221 & -3.014 & -1.075 \\
150 & -3.761 & -2.932 & -0.386 \\
152 & -3.922 & -2.957 & -0.637 \\
154 & -3.678 & -2.859 & -0.390 \\
156 & -3.716 & -2.832 & -0.515 \\
158 & -3.832 & -2.824 & -0.717 \\
\hline \hline
\end{tabular}


calculations include the semimagic nucleus ${ }^{144} \mathrm{Sm}$, for which the BCS calculation leads to a normal solution with no pairing correlation energy. In contrast, the projected BCS calculation leads to substantial pairing correlations in the ground state. That number projection is critical in mean-field treatments of semimagic nuclei is well known from other calculations [12]. The exact treatment of pairing leads to a further lowering of the energy of the ground state of the system, by $0.3 \mathrm{MeV}$.

For non-semi-magic nuclei, the effect on the pairing correlation energy of the exact solution is even more pronounced. While PBCS gives a significant lowering of the energy due to number projection, it misses about $1 \mathrm{MeV}$ of the exact correlation energy. Considering the extensive recent efforts to carry out systematic microscopic calculations of nuclear masses using mean-field methods [13], we feel that this effect may be quite meaningful. While a renormalization of the strength of the pairing interaction could accommodate these important corrections, it might not be possible to resort to a simple renormalization recipe when dealing with more general effective interactions, such as the Gogny force, where the particle-hole and particle-particle channels are consistently treated in a full HFB calculation.

Our results for the $\mathrm{Sm}$ isotopes are consistent with those from studies of ultrasmall superconducting grains $[14,15]$. There, the quantum phase transition from a superconducting to a normal metal predicted by BCS and PBCS disappeared after including the pairing fluctuations by means of exact solution of the BCS model, and this could not be realized by simply renormalizing the pairing strength in BCS or PBCS approximation. Moreover, the PBCS wave function displayed a strange behavior in the transitional region as compared with the smooth behavior of the exact wave function [16].

A second important feature of Cooper pairing is the condensate fraction, namely the fraction of pairs of the whole system that are correlated. Analysis of the off-diagonal long-range order (ODLRO) that characterizes superconductors and superfluids led Yang [6] to a definition of the condensate fraction, $\lambda$, in terms of the single macroscopic eigenvalue of the two-body density matrix. For a homogeneous system of two spin fermion species in the thermodynamic limit, $\lambda$ is given by

$$
\lambda=\int d^{3} \mathbf{r}_{1} d^{3} \mathbf{r}_{2}\left|\left\langle\psi_{\downarrow}\left(\mathbf{r}_{1}\right) \psi_{\uparrow}\left(\mathbf{r}_{2}\right)\right\rangle\right|^{2}=\frac{1}{M} \sum_{k>0} u_{k}^{2} v_{k}^{2}
$$

This definition is not appropriate for finite Fermi systems, however, where several eigenvalues of the two-body density matrix are of the same order. We modify it, therefore, by excluding from the two-body density matrix the amplitude of finding two uncorrelated fermions. More specifically, our prescription for finite systems is to evaluate the matrix elements of the operator

$$
\lambda=\frac{1}{M(1-M / L)} \sum_{k, k^{\prime}=1}^{L}\left\langle c_{k}^{\dagger} c_{\bar{k}^{\prime}}^{\dagger} c_{\bar{k}^{\prime}} c_{k}\right\rangle-\left\langle c_{k}^{\dagger} c_{k}\right\rangle\left\langle c_{\bar{k}^{\prime}}^{\dagger} c_{\bar{k}^{\prime}}\right\rangle
$$

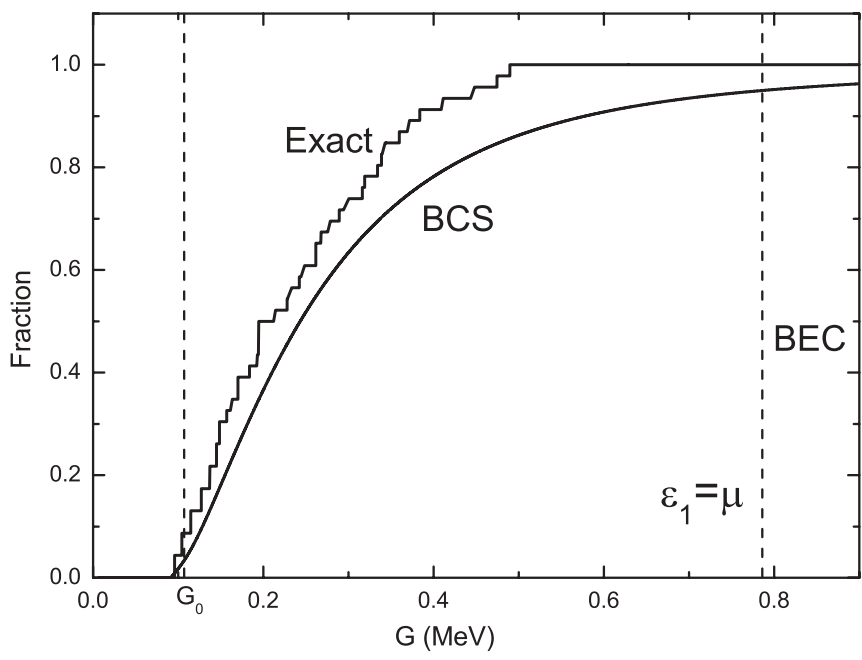

FIG. 1. The modified Yang prescription for the BCS treatment of pairing (smooth curve) and the alternative prescription discussed in the text (sawtooth curve) for the exact Richardson treatment. $G_{0}=$ $0.106 \mathrm{MeV}$ denotes the physical value of the pairing strength and $\epsilon_{1}=\mu$ denotes the strength at which the whole system binds.

where $L$ is the total number of doubly-degenerate, canonically conjugate pair states $k, \bar{k}$.

In BCS approximation, the modified Yang prescription leads to a condensate fraction

$$
\lambda_{\mathrm{BCS}}=\frac{1}{M(1-M / L)} \sum_{k=1}^{L} u_{k}^{2} v_{k}^{2} .
$$

We have calculated this quantity for the BCS solutions obtained for ${ }^{154} \mathrm{Sm}$ as a function of the pairing strength $G$ and plot the results as the smooth curve in Fig. 1.

An alternative prescription for the condensate fraction from the exact Richardson solution was proposed in [5] and shown to more properly reflect the properties of a superfluid system as it undergoes the crossover from BCS to BEC. In particular, this new prescription gives a fully condensed state at the change of sign of the chemical potential where the whole system becomes bound. This prescription, however, requires knowledge of the properties of the precise Cooper pairs in the problem, not an average over the whole system as provided by the BCS or PBCS approximation Eqs. (3) and (4). The Richardson ansatz (5) is ideally suited for this as it provides an exact wave function for each individual Cooper pair. One has to simply distinguish which pairs are correlated and which are not. As previously discussed, a correlated pair is characterized by a pair energy $e_{\alpha}$ that is far enough away in the complex plane from any particular $2 \epsilon_{k}$. We therefore propose the following practical definition for the condensate fraction: It is the fraction of pair energies which in the complex energy plane lie further from any unperturbed single-pair energy, $2 \epsilon_{k}$, than the mean single-particle level spacing.

We now return to a discussion of the condensate fraction, as plotted in Fig. 1 for ${ }^{154} \mathrm{Sm}$ as a function of the pairing strength $G$. In addition to the results based on the pair correlator, as discussed earlier, we also plot (in the sawtooth curve) the 

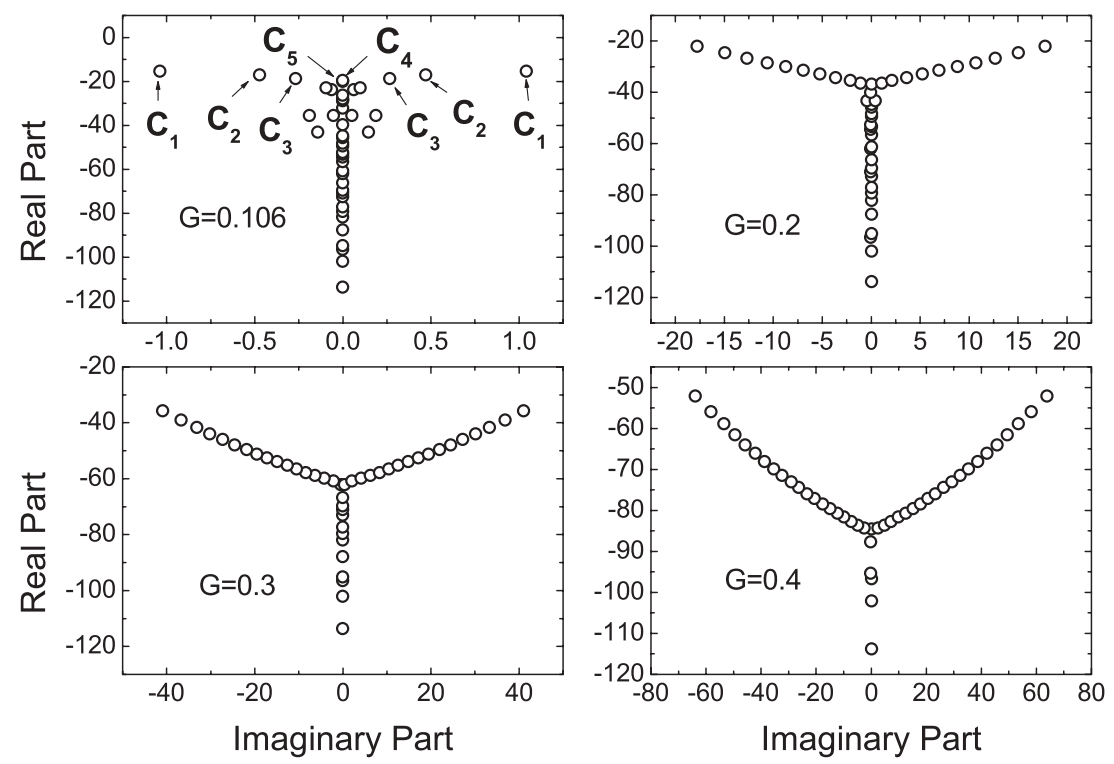

FIG. 2. Pair energies (in $\mathrm{MeV}$ ) for the exact Cooper pairs that emerge from four calculations of the ${ }^{154} \mathrm{Sm}$ isotope. $G=0.106 \mathrm{MeV}$ is the physical value of the pairing strength. In that panel, we denote the most collective pairs as $\mathrm{C}_{i}$, for subsequent notational purposes. results that derive from the exact Richardson solution using the prescription just described. To illustrate how these latter results emerge, we show in Fig. 2 the associated pair energies for four values of $G$ in ${ }^{154} \mathrm{Sm}$, ranging from the physical value of $G=0.106 \mathrm{MeV}$ to a fairly strong pairing strength of $G=0.4 \mathrm{MeV}$. In ${ }^{154} \mathrm{Sm}$ the mean level spacing between the Hartree Fock single-particle levels is roughly $0.5 \mathrm{MeV}$, both around the Fermi surface and far from it. For $G=$ $0.106 \mathrm{MeV}$, most of the pair energies lie very near the real axis and quite close to at least one unperturbed single-pair energy, $2 \epsilon_{k}$. Two of them (which form a complex conjugate pair) extend about $1 \mathrm{MeV}$ in the complex plane, while another two are marginally collective, lying roughly $0.5 \mathrm{MeV}$ from the closest $2 \epsilon_{k}$. The two most collective pairs, denoted $\mathrm{C}_{1}$ in the figure, each have a real energy of $-15.55 \mathrm{MeV}$, which is roughly twice the energy of the single-particle levels just below the Fermi surface. This suggests that the first pairs that become collective are indeed those built out of the valence orbits. As $G$ increases, we see a gradual increase in the number of collective pairs, which form an arc in the complex plane. As can be seen from Fig. 1, by a pairing strength of roughly $0.5 \mathrm{MeV}$ all of the pairs of the system are correlated giving a condensate fraction of 1 , even though the BEC regime has not yet been reached. The BEC limit is realized when the chemical potential $\mu$ crosses the lowest single-particle energy $\epsilon_{1}$ at $G=0.788$ for ${ }^{154} \mathrm{Sm}$. At this point all pairs are bound. However, the revised Yang prescription (9) fails to predict a complete condensate at this point, in the same way as it fails to do so in the homogeneous case [5].

The Richardson prescription for Cooper pairs also gives rise to a different interpretation of their internal structure. In Fig. 3, we compare the square of the wave function for the most correlated Cooper pairs in ${ }^{154} \mathrm{Sm}$, i.e., those whose pair energies lie farthest from any unperturbed single-pair energy, with the square of the pair correlator wave function obtained from the BCS calculation. All wave functions are plotted versus the order of the single-particle states to make clear the relevant mixing of configurations in each pair. The pair labels in the figure $\left(\mathrm{C}_{1}\right.$ through $\left.\mathrm{C}_{5}\right)$ refer to corresponding labels in the upper left panel of Fig. 2. $\mathrm{C}_{1}$ refers to the two most collective pairs, namely those that are farthest from any unperturbed single-particle pair. Being complex conjugate pairs, both have exactly the same absolute square of their wave function and thus we only show one in the figure. $\mathrm{C}_{2}$ refers to the next two most collective pairs, which as noted earlier are marginally collective according to our prescription. $\mathrm{C}_{3}$ refers to the next two most collective pairs after $\mathrm{C}_{2}$, which according to the prescription given above involve perturbative mixing of configurations and are not collective. $\mathrm{C}_{4}$ and $\mathrm{C}_{5}$, the following pairs in descending collective order, have real pair energies and involve almost pure single-particle configurations.

From the figure, we see that the pair correlator wave function is spread over several single-particle configurations and peaked at the 47th single-particle level, just beyond the Fermi energy $\left({ }^{154} \mathrm{Sm}\right.$ has 46 neutron pairs). In contrast,

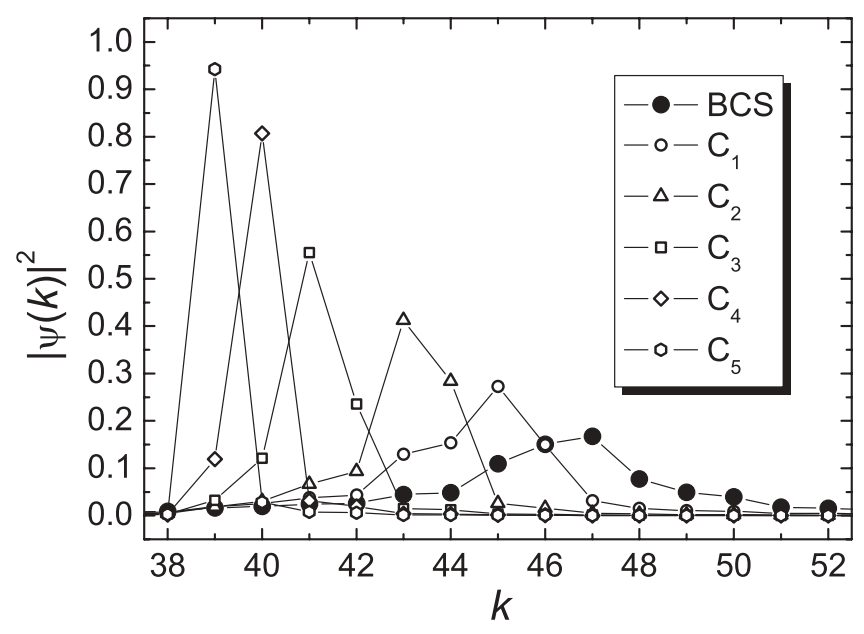

FIG. 3. Square of the wave function of the most collective Cooper pairs in ${ }^{154} \mathrm{Sm}$ (denoted $\mathrm{C}_{1}, \mathrm{C}_{2}, \mathrm{C}_{3}, \mathrm{C}_{4}$, and $\mathrm{C}_{5}$ ) and the pair correlator (BCS) versus the single-particle levels. 
the most highly correlated Cooper pair wave function $\mathrm{C}_{1}$ is somewhat narrower (less collective) and is peaked slightly within the Fermi sphere. The less-collective Cooper pairs, $\mathrm{C}_{2}$ through $\mathrm{C}_{3}$, are peaked progressively further inside the Fermi sphere and are progressively narrower. From this figure, we conclude that the size of even the most collective Cooper pairs in coordinate space will be larger than the size of the pair correlator, as was already demonstrated in the weak coupling BCS regime of cold atomic gases [5]. Investigations [7,8] on the size of the pair correlator in spherical nuclei concluded that it is unexpectedly small in the nuclear surface (2-3 fm). The present calculations would suggest that the actual size of the few highly collective Cooper pairs is larger than the typical size of the pair correlations in the nuclear medium. Furthermore, as is also evident from the figure, less bound pairs get progressively closer to a particular $2 \epsilon_{k}$ and the corresponding Cooper pair wave function is less collective, i.e., more narrow in energy space, and peaked at this particular configuration.

In this work, we have studied the role of Cooper pairing in atomic nuclei, focusing on a realistic description of the even $\mathrm{Sm}$ isotopes. We assume that the mean field is given by the self-consistent HF solution from coupled $\mathrm{HF}+\mathrm{BCS}$ calculations, and then consider how the effects of pairing on that mean field would be modified at several levels of improved treatment. We consider both the projected BCS approximation and an exact treatment based on Richardson's solution of the pairing problem. Several important points emerged. On the one hand, even though PBCS approximation gives a significant gain in binding energy over ordinary BCS, it still fails to capture a sizable component, typically of order $1 \mathrm{MeV}$. This might have important implications in efforts to derive nuclear masses from a microscopic approach. Second, we discussed a new and improved prescription for identifying the fraction of the pairs in a nucleus that are collective, which can only be realized when the properties of the various Cooper pairs in the problem are treated separately. This new prescription suggests that a slightly larger number of pairs are collective when compared to the more usual prescription based on Yang's definition of the condensate fraction. Furthermore, it suggests that the few collective Cooper pairs that arise in real nuclei, being individually less collective than the pair correlator, would be spatially more spread out.

The Richardson solution, as generalized in Ref. [17], can be obtained for integrable pairing Hamiltonians only. It is possible, however, to use the Richardson ansatz (5) in a variational treatment of general nonintegrable pairing Hamiltonians. The pair energies would play the role of variational parameters in a generalized Pfaffian pairing wave function [18], making it possible to treat pair correlations more precisely for realistic nuclear systems.

We acknowledge fruitful discussions with N. Sandulescu, P. Schuck, and W. Nazarewicz. This work was supported in part by the Spanish DGI under grants FIS2005-00640 and FIS2006-12783-C03-01, in part by the U.S. National Science Foundation under grant No. 0553127, and in part by UBACYT X-053, Carrera del Investigador Científico and PIP-5287 (CONICET-Argentina). One of the authors (S.P.) wishes to acknowledge the generous support and hospitality of the CSIC in Madrid where much of his contribution to the work was carried out.
[1] L. N. Cooper, Phys. Rev. 104, 1189 (1956).

[2] J. Bardeen, L. N. Cooper, and J. R. Schrieffer, Phys. Rev. 108, 1175 (1957).

[3] A. Bohr, B. R. Mottelson, and D. Pines, Phys. Rev. 110, 936 (1958).

[4] R. W. Richardson, Phys. Lett. 3, 277 (1963); Phys. Rev. 141, 949 (1966).

[5] G. Ortiz and J. Dukelsky, Phys. Rev. A 72, 043611 (2005).

[6] C. N. Yang, Rev. Mod. Phys. 34, 694 (1962).

[7] M. Serra, A. Rummel, and P. Ring, Phys. Rev. C 65, 014304 (2001).

[8] M. Matsuo, K. Mizuyama, and Y. Serizawa, Phys. Rev. C 71, 064326 (2005); N. Pillet, N. Sandulescu, and P. Schuck, nuclth/0701086.

[9] K. Dietrich, H. J. Mang, and J. H. Pradal, Phys. Rev. 135, B22 (1964).
[10] F. Dominguez, C. Esebbag, and J. Dukelsky, J. Phys. A: Math. Gen. 39, 11349 (2006).

[11] S. Rombouts, D. Van Neck, and J. Dukelsky, Phys. Rev. C 69, 061303(R) (2004).

[12] M. V. Stoitsov, J. Dobaczewski, R. Kirchner, W. Nazarewicz, and J. Terasaki, nucl-th/0610061.

[13] D. Lunney, J. M. Pearson, and C. Thibault, Rev. Mod. Phys. 75, 1021 (2003).

[14] J. Dukelsky and G. Sierra, Phys. Rev. Lett. 83, 172 (1999).

[15] M. A. Fernandez and J. L. Egido, Phys. Rev. B 68, 184505 (2003).

[16] J. Dukelsky and G. Sierra, Phys. Rev. B 61, 12302 (2000).

[17] J. Dukelsky, C. Esebbag, and P. Schuck, Phys. Rev. Lett. 87, 066403 (2001).

[18] M. Bajdich, L. Mitas, L. K. Wagner, and K. E. Schmidt, condmat/0610850. 